\title{
Wavelet-based action potential detector for the extracellular neural signal with low signal-to-noise ratio
}

\author{
Kyung Hwan Kim, Sung June Kim* \\ Human-computer interaction laboratory, Samsung advanced institute of technology, Yongin, Korea \\ *School of electrical engineering and computer science, Seoul National University, Seoul, Korea
}

\begin{abstract}
We develop a wavelet-based action potential detection method to lower the level of signal-to-noise ratio (SNR) required for successful detection. It is based on point-wise product of wavelet coefficients of several scales, and effectively performs the role of combination of matched filters. We show its performance for various neural signal recordings. Detection of action potential from extracellular neural signal can be difficult due to the low SNR and high similarity between the target signal and noise. In previous studies of experimental neurophysiology using the extracellular recording, only the action potentials with sufficiently large amplitude have been gathered and analyzed. We compared its performance with those of the linear optimal filter and the Teager energy operator, and showed the superiority of the proposed detector over them. Test was performed for various SNR values and degrees of spectral similarity between the target signal and the noise.
\end{abstract}

Keywords - action potential detection, extracellular recording, wavelet transform, matched filter

\section{INTRODUCTION}

Because the information of the nervous system is encoded as firing frequency or firing time of action potential (AP), the first procedure to interpret the neuronal signal is detection of the APs. However, we cannot find much study on neural spike detection itself from the literature. We often confront the cases when signal-to-noise ratio (SNR) of the extracellular recording is so poor as to prohibit neural spike detection using simple thresholding. Moreover the characteristics of the background noise can be very similar to those of the target signal. Satisfactory enhancement of the SNR using conventional signal processing techniques such as bandpass filtering, becomes difficult.

In this paper, we present a method of action potential detection for various situations of extracellular neural signal recordings. Our detection method is based on the point-wise product of wavelet transform coefficients over several successive scales. We show that it performs essential function of the matched filter detection without a priori knowledge on the target signal and noise, and shows better performance in real noisy situations. We also compare its performance with those of AP detection methods using linear optimal filter and Teager energy operator (TEO).

\section{DETECTION METHOD}

The AP is distinguished by instantaneous rise in amplitude and frequency. Essentially, the wavelet transform carries out a correlation analysis between the input signal and the translated and dilated version of a reference signal called mother wavelet. Hence its output yields a local maximum when the input signal most closely resembles the analysis template (the wavelet function). The wavelet basis functions have 'spiky' (biphasic or triphasic and so on) waveform. Thus they are similar in shape to the APs, and can act as an effective approximate of the matched filter for AP at some particular scales, even though we do not know the exact waveform of the target signals. We further enhance its performance by combining information obtained at several scales. For the fast computation of the wavelet coefficient, the scale is limited to the dyadic ones, and the translation is limited to the integer multiples of the scale. This is called the discrete wavelet transform (DWT) [1]. The DWT can be performed by a bank of discrete-time filters and necessary computation time is much shorter.

Now we describe procedure of our AP detector. First, the wavelet coefficients and their absolute values are calculated for 5 dyadic scales, i.e. $2^{1} \sim 2^{5}$, and the scale where the absolute yields maximum value (this scale is called $j_{\max }$ ) is selected. After that as shown below in equation (5), $P(n)$, product of the wavelet coefficients over three consecutive scales up to $j_{\max }$, is calculated for all the time samples, $n$, as follows:

$$
P(n)=\prod_{j=j_{\max }-2}^{j_{\max }}\left|W\left(2^{j}, n\right)\right|
$$

Three successive scales up to $j_{\max }$ is appropriate for most cases of AP detection. Inclusion of too large or too small scales decreased the peaks due to the APs because the correlation between the analysis template (dilated version of the wavelet basis) and the target signal (AP) is small for those scales. $P(n)$ is smoothed by being convolved with the Bartlett window in order to eliminate the spurious peaks due to the cross terms and background noise. Length of the window is determined empirically and found to be suitable when it is about half of the duration of the target signal. Smoothed version of $P(n)$ is compared to the threshold to determine the position of AP peak.

Fig. 1 shows rationale for the DWT detector. As denoted by the thick line, the peaks in the wavelet transform coefficients due to the transient in the input signal appear at the same time point over successive scales, while due to the background noise (denoted by ' $\sqrt{ }$ ') is prominent only on single scale. By calculating point-by-point product, we can enhance the signal peaks and suppress the noise peaks.

\section{RESULTS}

We tested performance of the proposed detector for various the degree of SNR, and the spectral similarity between the signal and noise. Test was performed on three experimental recordings: from somatosensory cortex of rat, from medial nerve of crayfish, and from the cutaneous nerve of the bullfrog 


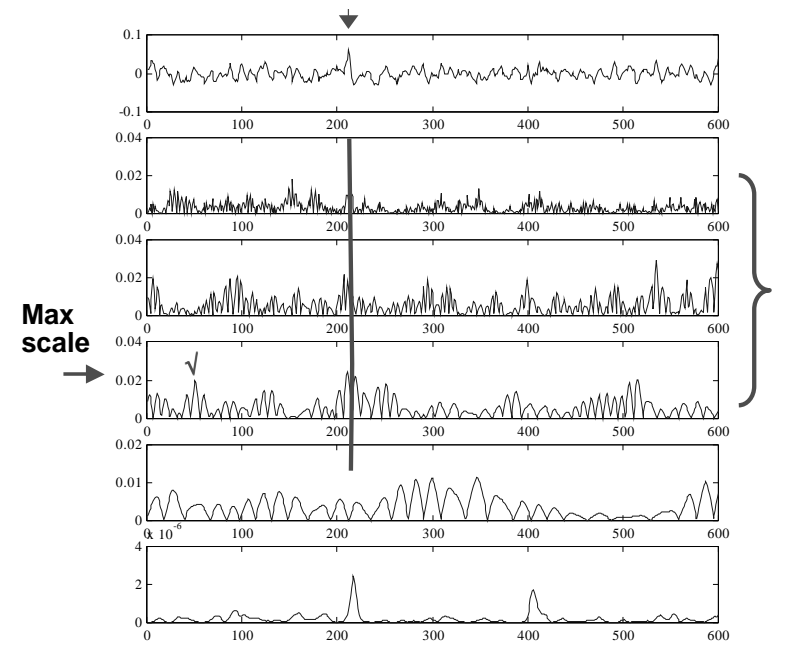

Fig. 1: Illustration of the rationale of the proposed detector.

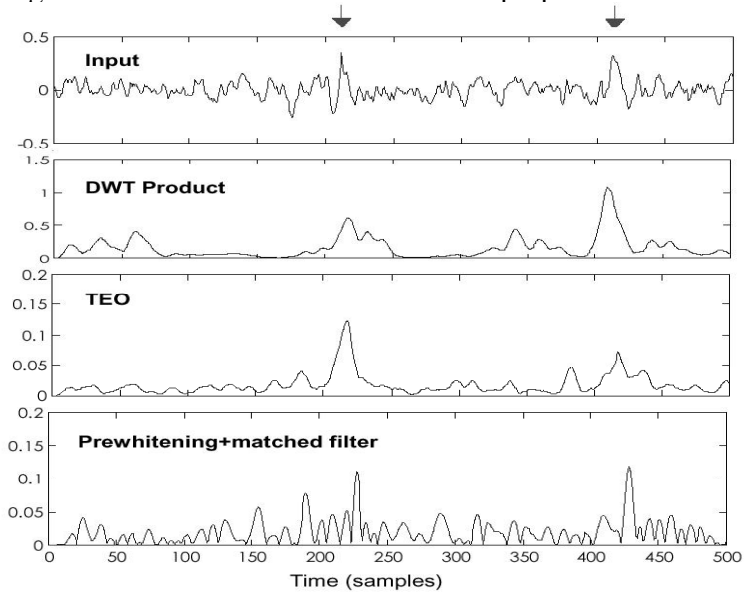

Fig. 2: Comparison of the signal quality after processing by the matched filter and proposed detectors

[2]. Time-series prediction technique was used for the modeling of the experimental background noise for the the arbitrary SNR's can be generated. We used 5th order AR model for the rat cortex and crayfish data, and 15th order AR model for the bullfrog data. Details of the experiments are described in [2], [3].

Fig. 2 shows the input waveform (recording from rat somatosensory cortex of rat, $\mathrm{SNR}=2.33$ ) and output waveforms from the proposed detector, and two other AP detectors. The arrows indicate the location of action potential. The TEO and (matched filter+prewhitening filter) yielded signal peaks sharper than the signal peaks obtained by the wavelet-based detector, and more spurious noise peaks. AP detector by TEO utilizes the characteristic of AP firing, i.e., instantaneous rise in frequency and amplitude [3]. It is not possible to judge whether the linearly optimal method (matched filter+prewhitening) is superior or inferior to the proposed detector from Fig. 2 alone.

We show more detailed comparison of detection performance by receiver operating characteristic (ROC). The ROC curve is obtained by plotting the $P_{D}$ (ratio of correct detection) as a function of $P_{F A}$ (ratio of false alarm). Each data

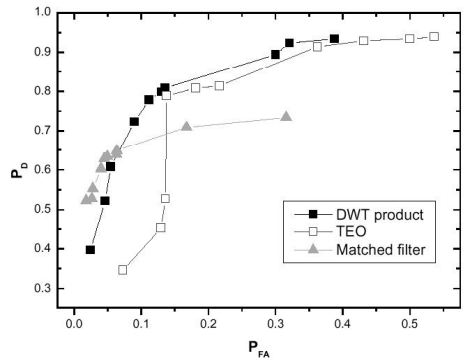

Fig. 3: Comparison of detection performance by ROC.

point in a curve corresponds to each different threshold value. Fig. 3 is result for the rat cortex data. The DWT detector and the TEO shows similar level of $P_{D}$ when high level of $P_{F A}$ is allowed, but the DWT detector showed superior performance for low level of $P_{F A}$. Both detectors were superior to the unrealizable matched filter with prewhitening. For the crayfish data the difference of performance between two detectors was larger. The TEO detector showed a little inferior performance than that of the matched filter for low $P_{F A}$. The proposed detector showed constantly higher performance than that of the matched filter. For the bullfrog data, both the proposed detector and TEO were not as good as the matched filter, however, performance of the DWT product detector was comparable to that of the matched filter.

\section{DISCUSSION AND CONCLUSION}

Detection of the action potential is a preliminary step which can determine the overall performance of the neural spike sorting system. In spite of this significance, studies on action potential detection have been rare. Only a few works on action potential detection can be found, most of them as a part of spike sorting work. The proposed detection method does not require the 'quantitative' information on the target signal and background noise; we made use of the fact that wavelet coefficients at several scales act as effective approximations of the matched filter and this is universal. Therefore, our methods have merit in that they are applicable for the general experimental cases where no preliminary quantitative information is available.

In conclusion, we suggested a method for the action potential detection from low SNR extracellular neural signal recording, and showed its superior performance by comparing with the prewhitening/matched filter detection.

\section{REFERENCES}

[1] S. Mallat and W. L. Hwang, "Singularity detection and processing with wavelets," IEEE Trans. Signal Processing, vol. 38, pp. 617-643, 1990

[2] K. H. Kim, "Methods for detection and classification of multiunit extracellular neural signal with high background noise," Ph. D. Dissertation, Seoul National University, Seoul, Korea, Feb. 2001

[3] K. H. Kim and S. J. Kim, "Neural spike sorting under Nearly $0 \mathrm{~dB}$ signal-to-noise ratio using nonlinear energy operator and artificial neural network classifier," IEEE Trans. Biomed. Eng., vol. 47, pp. 1406-1411, 2000 